\title{
THE JET/COUNTERJET INFRARED SYMMETRY OF HH 34 AND THE SIZE OF THE JET FORMATION REGION
}

\author{
A. C. Raga ${ }^{1}$, A. Noriega-Crespo ${ }^{2}$, V. Lora $^{3}$, K. R. Stapelfeldt ${ }^{4}$, And S. J. Carey ${ }^{2}$ \\ ${ }^{1}$ Instituto de Ciencias Nucleares, Universidad Nacional Autónoma de México, Ap. 70-543, 04510 D.F., Mexico \\ ${ }^{2}$ SPITZER Science Center, California Institute of Technology, CA 91125, USA \\ ${ }^{3}$ Astronomisches Rechen-Institut Zentrum für Astronomie der Universität Heidelberg, Mönchhofstr. 12-14, 69120 Heidelberg, Germany \\ ${ }^{4}$ Jet propulsion Laboratory, California Institute of Technology, MS 183-900, 4800 Oak Grove Drive, Pasadena, CA 91109, USA \\ Received 2010 December 14; accepted 2011 January 12; published 2011 March 4
}

\begin{abstract}
We present new Spitzer IRAC images of the HH 34 outflow. These are the first images that detect both the knots along the southern jet and the northern counterjet (the counterjet knots were only detected previously in a long-slit spectrum). This result removes the problem of the apparent coexistence of a large-scale symmetry (at distances of up to $\sim 1 \mathrm{pc}$ ) and a complete lack of symmetry close to the source (at distances of $\sim 10^{17} \mathrm{~cm}$ ) for this outflow. We present a quantitative evaluation of the newly found symmetry between the HH 34 jet and counterjet, and show that the observed degree of symmetry implies that the jet production region has a characteristic size $<2.8 \mathrm{AU}$. This is the strongest constraint yet derived for the size of the region in which $\mathrm{HH}$ jets are produced.
\end{abstract}

Key words: circumstellar matter - Herbig-Haro objects - infrared: ISM - ISM: individual objects (HH 34) - ISM: jets and outflows - stars: formation

Online-only material: color figures

\section{INTRODUCTION}

HH 34 is one of the Herbig-Haro (HH) objects in Herbig's catalog (Herbig 1974). It jumped into the limelight with the paper of Reipurth et al. (1986), who showed that the HH object (HH 34S) had a bow-shaped morphology and a jet-like association of aligned knots (pointing toward the apex of the bow shock). Later observations showed the existence of a northern counterpart to $\mathrm{HH} 34 \mathrm{~S}(\mathrm{HH} 34 \mathrm{~N})$ and of a series of bipolar bow shock pairs at larger distances from the outflow source (Bally \& Devine 1994; Eislöffel \& Mundt 1997; Devine et al. 1997). There is a wealth of observations of this outflow, including images, spectrophotometry, radial velocity, and proper motion measurements at optical (see, e.g., Heathcote \& Reipurth 1992; Eislöffel \& Mundt 1992; Morse et al. 1992, 1993; Reipurth et al. 2002; Beck et al. 2007) and IR wavelengths (Stapelfeldt et al. 1991; Stanke et al. 1998; Reipurth et al. 2000). These observations show that the HH 34 outflow (at a distance of $\approx 417$ pc; see Menten et al. 2007) has a plane of the sky velocity of $\approx 150 \mathrm{~km} \mathrm{~s}^{-1}$ and propagates at an angle of $\approx 30^{\circ}$ from the plane of the sky (the southern lobe being directed toward the observer).

The flow itself is driven by HH 34 IRS, a Class I protostar surrounded by a relatively large $(\sim 1000$ AU radius $)$ circumstellar disk (Stapelfeldt \& Scoville 1993; Anglada et al. 1995). The possible coupling of the disk and outflow has motivated the search for signatures of internal rotation within the jet as a consequence of the transfer of angular momentum from the rotating disk into the highly collimated jet (Coffey et al. 2011).

In spite of the clear bipolar symmetry of the bow shock pairs (extending to $\sim 1.5 \mathrm{pc}$ from the source; see Devine et al. 1997), observations extending over $\sim 2$ decades did not detect a northern counterpart for the chain of aligned knots extending $\sim 30^{\prime \prime}$ southward from the HH 34 source. This situation changed with the paper of García López et al. (2008), who obtained IR (1.6 and $2.1 \mu \mathrm{m}$ ) long-slit spectra in which the emission of the northern counterjet was finally detected. These authors note that the emission of the counterjet has intensity peaks at positions (i.e., distances from the source) which approximately coincide with the knots along the southern jet.

In this Letter, we present new Spitzer IRAC images of HH 34. These images show the southern jet and northern counterjets with comparable intensities, and with a surprising degree of symmetry. The observations are described in Section 2. In Section 3, we present an image of the central region of the HH 34 outflow, quantitatively evaluate the degree of symmetry between the jet and the counterjet, and discuss the implications of the results for the ejection mechanism that has produced the outflow. The results are summarized in Section 4.

\section{OBSERVATIONS}

The observations of HH 34 are part of our original Spitzer Space Telescope (Werner et al. 2004) General Observer (GO) program 3315 (PI: A. Noriega-Crespo) obtained with both the infrared camera IRAC (Fazio et al. 2004) and the infrared photometer MIPS (Rieke et al. 2004) in 2005 March 28. The data have been recovered from the Spitzer Legacy Archive and the quality of the final images (Post Basic Calibrated Data or Post-BCD; S18.7 products) is outstanding, so that no further processing was required. In this study, we present the IRAC observations obtained in the four channels $(1,2,3,4)=(3.6$, $4.5,5.8,8.0 \mu \mathrm{m}$ ) covering a field of view of $\sim 30^{\prime} \times 30^{\prime}$ (the result of a $6 \times 6$ array map with a $260^{\prime \prime}$ stepsize) and with a total integration time per pixel of $30 \mathrm{~s}$. The final images are sampled with 0 "' 6 per pixel, nearly one-third of standard $\sim 2^{\prime \prime}$ IRAC angular resolution.

Figure 1 shows a three color image of HH 34 using channels 1,2 , and 3. Like with other protostellar outflows observed with IRAC (see, e.g., Noriega-Crespo et al. 2004; Looney et al. 2007; Tobin et al. 2007; Ybarra \& Lada 2009), Channel 2 recovers the strongest jet emission, since its bandpass (4 to $5 \mu \mathrm{m}$ ) includes three relatively bright pure rotational $\mathrm{H}_{2}$ emission lines, $0-0$ $\mathrm{S}(9) 4.69,0-0 \mathrm{~S}(10) 4.41$, and $0-0 \mathrm{~S}(11) 4.18 \mu \mathrm{m}$; the jet is well detected in Channel 3 as well, where another couple of $\mathrm{H}_{2}$ lines 


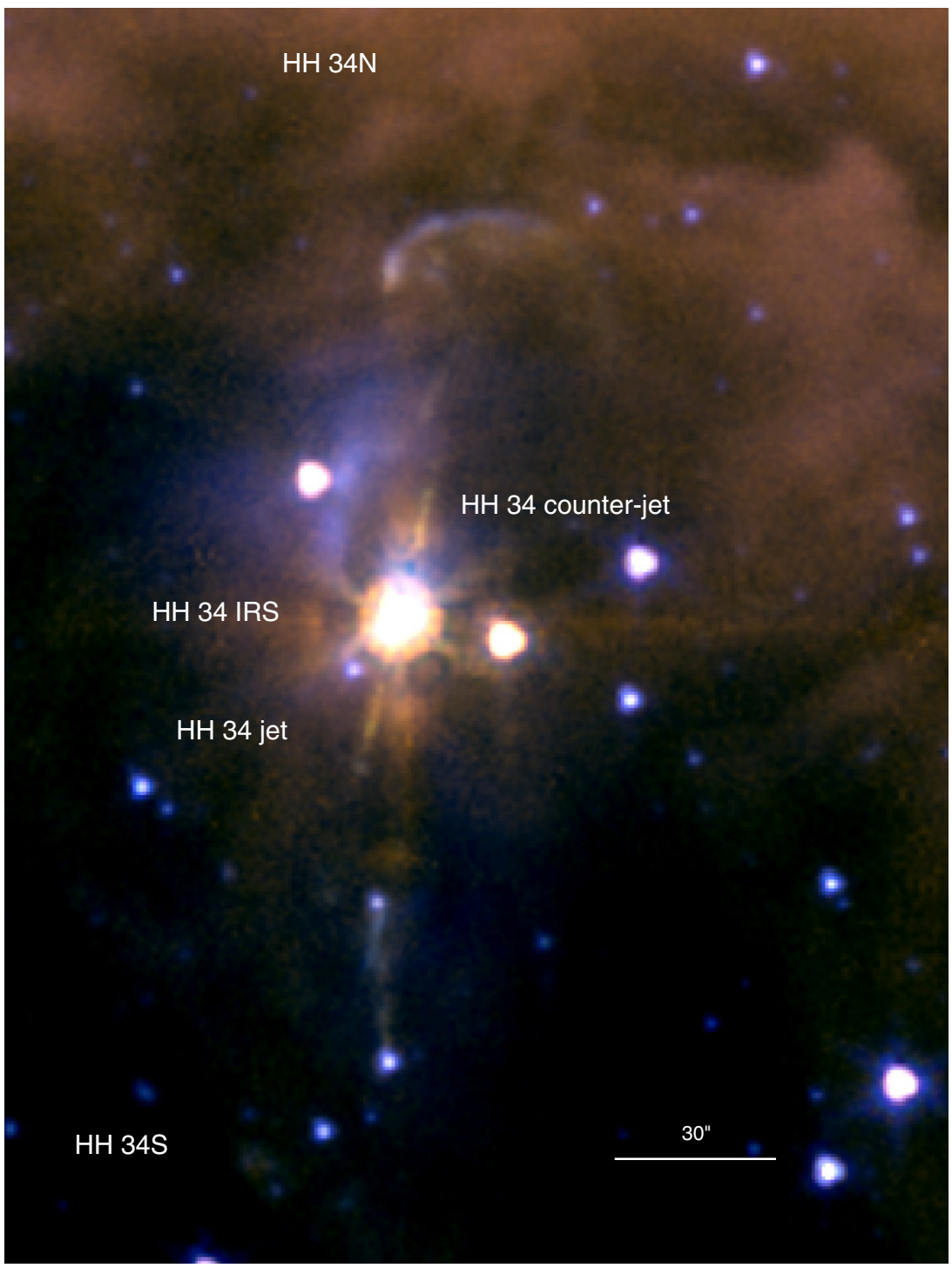

Figure 1. Section of the HH 34 IRAC map $\left(\sim 3^{\prime} \times 4^{\prime}\right)$ centered on the HH 34 IRS source using the four channels 3.6 (blue), $4.5+5.8$ (green), and $8 \mu \mathrm{m}$ (red). North is up and east to the left. The image shows the counterjet as well as a wider infrared bow shock about $20^{\prime \prime}$ south of the optical HH $34 \mathrm{~N}$ main bow shock. Indeed both $\mathrm{HH} 34 \mathrm{~S}$ and $34 \mathrm{~N}$ lie within the image, but are barely visible. Other extended emission structures (e.g., the north-south, jet-like structure south of HH 34 IRS) seen in the map might be associated with other outflows in the region.

(A color version of this figure is available in the online journal.)

are found $(0-0 \mathrm{~S}(6) 6.11$ and 0-0 S(7) $5.51 \mu \mathrm{m}$; see De Buizer \& Vacca 2010).

\section{THE JET/COUNTERJET SYMMETRY}

In Figure 2, we show the $4.5+5.8 \mu \mathrm{m}$ image of the region around the source of $\mathrm{HH} 34$ rotated so that the outflow axis is parallel to the ordinate. This image shows a surprising symmetry between the southern jet (detected in optical images) and the northern counterjet. We have defined a 7 pixel (4".2) wide, rectangular box aligned with the HH 34 axis, within which we subtract the background emission (defined as a linear interpolation between the pixels immediately outside of the long edges of the box). The result of this background emission subtraction is shown on the right-hand side of Figure 2, in which the knot structure along the $\mathrm{HH} 34$ jet and counterjet is seen more clearly.

We have carried out paraboloidal fits to the emission peaks of the knots along the outflow axis, determining the positions of the peaks with an error of $\approx 0.2$ pixels $=0$ ' 12 . Through this procedure, we obtain the positions $x_{j}$ and $x_{c j}$ of the seven knots along the jet and the counterjet (respectively). The position of the source is not so well determined, because the image of the source is partially saturated and has a complex point-spread function. Because of this, we have estimated the position of the source as the average of the coordinates of the seven knots along the jet and the counterjet. A paraboloidal fit to the emission of the region around the source actually results in a similar position (with offsets of $\approx 0.2$ pixels along and $\approx 1$ pixel across the jet axis with respect to the average position of the ensemble of knots).

In Figure 3, we plot the positions $x_{c j}$ of the consecutive knots along the counterjet as a function of the positions $x_{j}$ of the corresponding knots along the jet. This figure shows the remarkable symmetry (with respect to the position of the source) of the knots along the jet and the counterjet.

In Figure 3, we also plot the offsets $\Delta x=\left|x_{j}-x_{c j}\right|$ (between the positions of the corresponding jet/counterjet knot pairs) as a 


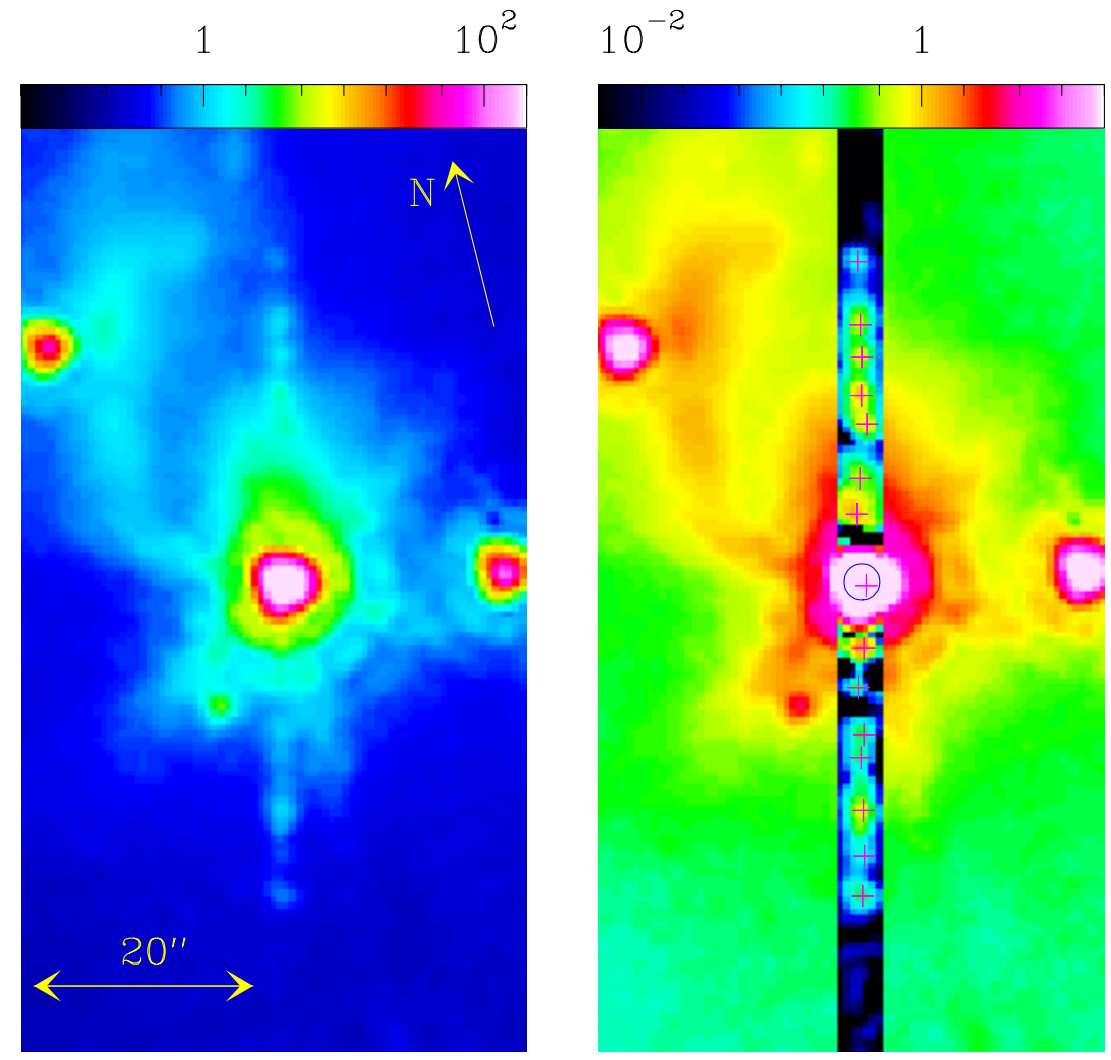

Figure 2. Central region of the 4.5+5.8 $\mu \mathrm{m}$ IRAC map (see Figure 1), rotated so that the HH 34 axis is parallel to the ordinate. The images correspond to the addition of the two bands and are displayed with the logarithmic color scales given (in $\mathrm{MJy} \mathrm{s}^{-1}$ ) by the bars on the top of each plot. The left plot shows the map, and the right plot shows a map in which a background subtraction has been applied within a rectangular box that includes the jet and the counterjet (see Section 3). The scale and orientation of the images are shown on the left-hand plot. On the right-hand plot, the red crosses show the positions of the outflow source and the jet/counterjet knots determined with the paraboloidal fits described in the text. The black circle shows the position of the source calculated as the average of the positions of the seven jet/counterjet knot pairs (see the text).

(A color version of this figure is available in the online journal.)

function of $x_{j}$. The first three knots have monotonically growing offsets, with $\Delta x=0$ '! $11 \rightarrow 0$ '! 44 (ranging from $\sim 1 \rightarrow 4$ times the measurement error, see above). The four knots further away from the source generally have larger offsets, with a top value $\Delta x=1$ 1".57 for the sixth knot out from the source. The resulting $\Delta x$ versus $x_{j}$ dependence therefore has low offsets close to the source and increasingly large (and more highly oscillating) values for larger distances from the source.

These results can be interpreted as follows. Let us first assume that the jet/counterjet knot pairs are ejected at the same time $\tau$, but with different velocities $v_{j}$ and $v_{c j}=v_{j}-\Delta v$ (for the jet and counterjet knots, respectively). If the knots are ballistic, at a time $t$ they will have positions $x_{j}=(t-\tau) v_{j}$ and $x_{c j}=(t-\tau)\left(v_{j}-\Delta v\right)$. One then obtains that the jet/counterjet knot offset $\Delta x_{v}$ (due to asymmetries in the jet/counterjet ejection velocities) is given by

$$
\Delta x_{v}=x_{j}-x_{c j}=\frac{\Delta v}{v_{j}} x_{j} .
$$

In other words, an ejection velocity asymmetry in the simultaneous ejection of a jet/counterjet knot pair results in a knot position asymmetry $\Delta x_{v}$ that increases linearly with distance from the source (as the knots travel away from the source).

Let us now assume that we have a pair of knots ejected with the same velocity $v_{j}$, but at times $\tau$ and $\tau+\Delta \tau$ (for the knot along the jet and the counterjet, respectively). For ballistic knots, at a time $t$ they would then be at distances $x_{j}=(t-\tau) v_{j}$ and $x_{c j}=(t-\tau-\Delta \tau) v_{j}$ from the source. The jet/counterjet knot offset $\Delta x_{\tau}$ (due to a time difference $\Delta \tau$ in the ejection time) is given by

$$
\Delta x_{\tau}=x_{j}-x_{c j}=\Delta \tau v_{j} .
$$

In other words, the position asymmetry $\Delta x_{\tau}$ does not change as the knots travel away from the source along the jet and counterjet.

For the case of jet/counterjet knot pairs ejected with a time difference $\Delta \tau$ and a velocity difference $\Delta v$, we therefore predict an asymmetry between the jet and counterjet knot positions of the form

$$
\Delta x=\left|\Delta x_{v}+\Delta x_{\tau}\right|=\left|\frac{\Delta v}{v_{j}} x_{j}+\Delta \tau v_{j}\right| .
$$

This relation gives the behavior of $\Delta x$ as a function of distance from the source $x_{j}$ (along the jet) for a given pair of knots as they travel down the jet/counterjet axis.

If we have a series of knot pairs (seven knot pairs in our HH 34 image), the values of $\Delta v / v_{j}$ and $\Delta \tau v_{j}$ will not necessarily be identical for all of the knot pairs. We can anyway attempt to fit a linear $\Delta x$ versus $x_{j}$ dependence to the observations.

At large enough distances from the source, Equation (3) is a straight line which goes through the origin (of the $\Delta x$ versus $x_{j}$ coordinates), with a slope of $\left|\Delta v / v_{j}\right|$. The $\Delta \tau v_{j}$ term (see Equation (3)) results in a scatter of points above and below (depending on the sign of $\Delta \tau$ ) this straight line. This scatter will 


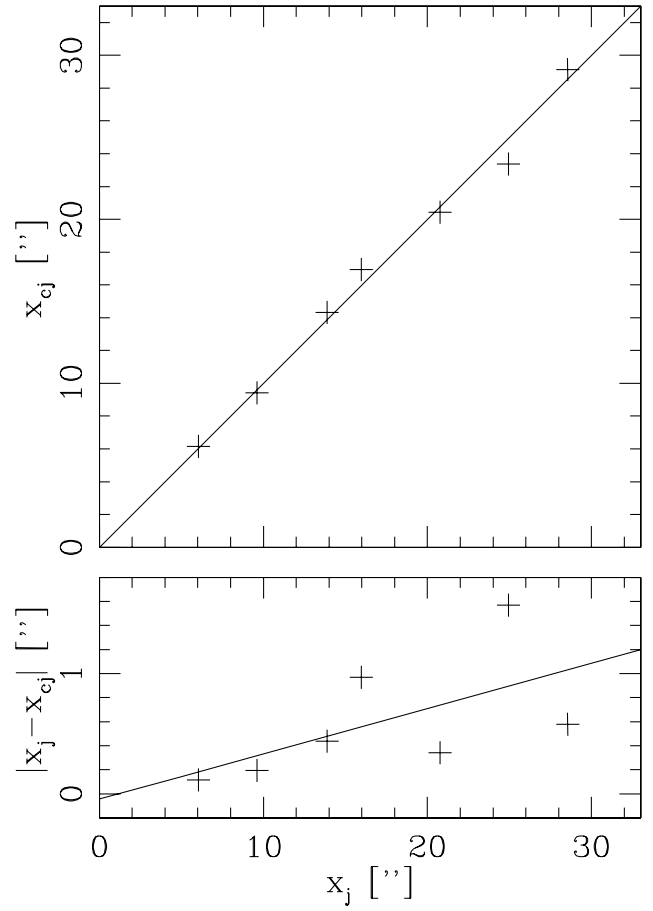

Figure 3. Top graph shows the distance from the source $x_{c j}$ of the knots along the northern counterjet as a function of the positions $x_{j}$ of the corresponding knots along the southern jet. The bottom plot shows the offsets $\Delta x=\left|x_{j}-x_{c j}\right|$ in the positions of the corresponding jet/counterjet knots as a function of distance $x_{j}$ from the source. The vertical size of the crosses in the $\Delta x$ vs. $x_{j}$ plot approximately corresponds to the error in the observational determinations of the $\Delta x$ values.

also have a contribution from the distribution of the $\left|\Delta v / v_{j}\right|$ values over the knot pairs.

Therefore, if we have a distribution of $\Delta \tau$ (over the different knot pairs) with a zero-mean velocity (i.e., with similar positive and negative $\Delta \tau$ values), we would expect to recover from the data a straight line that still goes through the origin. The slope of this line would represent an average of $\left|\Delta v / v_{j}\right|$ over the ensemble of knot pairs (see Equation (3)). The value of the determined error for the $x_{j}=0$ interception point of the straight line would give us an upper limit to the root mean square average of $\Delta \tau v_{j}$.

Fitting a least-squares linear dependence to the observed offsets, we obtain

$$
v_{j} \Delta \tau=(-0.04 \pm 0.36)^{\prime \prime} ; \quad \frac{\Delta v}{v_{j}}=0.038 \pm 0.019 .
$$

We therefore obtain an estimate for the average time offset of basically 0 (as expected), an upper limit for its root mean square value of $0.36 / v_{j}$, and a velocity asymmetry with an average modulus of approximately $(0.04 \pm 0.02) v_{j}$.

If we consider a distance of $417 \mathrm{pc}$ (to $\mathrm{HH} 34$ ) and that the knots have a plane of the sky velocity $v_{j} \approx 150 \mathrm{~km} \mathrm{~s}^{-1}$, we then obtain

$$
\Delta \tau \leqslant 4.5 \mathrm{yr} ; \quad \Delta v=(5.7 \pm 2.8) \mathrm{km} \mathrm{s}^{-1} .
$$

The maximum value of $\Delta \tau$ that we have determined in this way from the observed jet/counterjet asymmetry of $\mathrm{HH} 34$ directly implies a maximum possible size for the region in which the ejection is produced. The gas at the foot of a stellar jet is observed to have temperatures of $\sim 10^{3} \mathrm{~K}$, implying a sound speed of $\approx 3 \mathrm{~km} \mathrm{~s}^{-1}$. The Alvén velocity in this region is expected to have similar values. In order to synchronize the jet/counterjet ejections to a time $\Delta \tau$, the region producing both outflows has to be able to communicate causally within this time interval. Therefore, waves propagating at a velocity $v_{s}$ (this could be the Alfvén or the sound velocity, both $\sim 3 \mathrm{~km} \mathrm{~s}^{-1}$, see above) should be able to travel the characteristic size $D$ of the jet formation region within a time $\Delta \tau$. This condition can be written as

$$
D \leqslant v_{s} \Delta \tau, \text { so } D \leqslant\left(\frac{v_{s}}{3 \mathrm{~km} \mathrm{~s}^{-1}}\right) 2.8 \mathrm{AU},
$$

where for the second inequality we have used the value of $\Delta \tau$ derived from the observed HH 34 jet/counterjet asymmetry (see Equation (5)) and the estimated sound speed of $3 \mathrm{~km} \mathrm{~s}^{-1}$.

\section{SUMMARY AND CONCLUSIONS}

We have obtained IRAC images of the region around the source of the HH 34 outflow (see Figure 1). These images show the presence of a southern jet and a northern counterjet (previously only detected in the IR long-slit spectrum presented by García López et al. 2008). The seven knots along the jet and the counterjet have a remarkable symmetry with respect to the position of the outflow source (see Figure 2).

We find that if we plot the offsets $\Delta x=\left|x_{j}-x_{c j}\right|$ (where $x_{j}$ is the distance from the source of a knot along the jet and $x_{c j}$ is the distance to the corresponding knot along the counterjet) as a function of the position $x_{j}$ of the knots, we obtain a general trend of larger $\Delta x$ values for increasing distances from the source (see Figure 3). This trend can be interpreted (on the basis of a simple, ballistic knot model) as the result of a time offset $\Delta \tau$ (between the ejection of the two knots in a given pair) and of a velocity difference $\Delta v$ (between the velocities of the knot pair).

From a fit to the observed $\Delta x$ versus $x$ relationship, we determine a velocity asymmetry of $\Delta v=(5.7 \pm 2.8) \mathrm{km} \mathrm{s}^{-1}$ and an upper limit for the time offset of the binary ejection of $\Delta \tau=4.5 \mathrm{yr}$. Interestingly, the velocity asymmetry estimated for the binary ejections is within a factor of $\sim 2$ of the estimates for the sound and Alfvén speeds at the base of an outflow from a young star. Actually, the estimates of the sound and Alfvén speeds lie within the error bars of the $\Delta v$ value determined from the HH 34 jet/counterjet knot asymmetries.

It is possible to use the upper limit of $\approx 4.5 \mathrm{yr}$ to derive an estimate $D \approx 2.8 \mathrm{AU}$ for the size of the jet production region. For example, if one considers models of magnetocentrifugally driven disk winds, this estimate would imply a characteristic radius for the Alfvén surface (in which the outflow becomes super-Alfvénic) of $D \approx 2.8 \mathrm{AU}$. This is an interesting constraint on models of the production of jets from young stars (for a recent discussion of jet production models, see the review of Ferreira 2008 and the papers in the book of García \& Ferreira 2010).

Another possible interpretation is that the $\approx 2.8 \mathrm{AU}$ radius (derived from the observations) corresponds to the outer radius of the region of the accretion disk which gives rise to the jet. This interpretation might be more comfortable in terms of magnetocentrifugally driven disk winds, because an Alfvén surface of $\sim 3 \mathrm{AU}$ radius might be too small.

Very Large Array observations of sources of HH outflows had provided a constraint of a maximum size of $\approx 50 \mathrm{AU}\left(0{ }^{\prime} \cdot 1\right.$ at the distance of Orion) on the size of the jet production region (see, e.g., the HH $1 / 2$ observations of Rodríguez et al. 2000). The upper limit of $\approx 2.8$ AU that we have derived above for the size of the formation region of the HH 34 jet is therefore the most stringent condition yet. This size corresponds to $\approx 7$ mas, a 
resolution that will only be approached in direct observations of an $\mathrm{HH}$ source by future efforts with the ALMA interferometer. Observations with large baseline near-IR interferometers (such as Keck-I or VLT-I) could possibly also resolve such a region, provided that the emission is strong enough to be detected.

To conclude, we should point out that the derived upper limit of $\approx 2.8$ AU for the jet formation region is derived from a simple, ballistic approximation for the motion of the knots along the HH 34 jet and counterjet. Of course, the observed asymmetry could partially arise from deviations from a purely ballistic motion. If this is the case, the size implied for the jet formation region would be even smaller.

This work is based in part on observations made with the Spitzer Space Telescope which is operated by the Jet Propulsion Laboratory, California Institute of Technology under NASA contract 1407. The work of A.R. and V.L. was supported by the CONACyT grants 61547, 101356, and 101975.

\section{REFERENCES}

Anglada, G., Estalella, R., Mauersberger, R., Torrelles, J. M., Rodríguez, L. F., Cantó, J., Ho, P. T. P., \& D’Alessio, P. 1995, ApJ, 443, 682

Bally, J., \& Devine, J. 1994, ApJ, 428, 65

Beck, T., Riera, A., Raga, A. C., \& Reipurth, B. 2007, AJ, 133, 1221

Coffey, D., Bacciotti, F., Chrysostomou, A., Nisini, B., \& Davis, C. 2011, A\&A, 526, A40

De Buizer, J. M., \& Vacca, W. D. 2010, AJ, 140, 196

Devine, D., Bally, J., Reipurth, B., \& Heathcote, S. 1997, AJ, 114, 2095

Eislöffel, J., \& Mundt, R. 1992, A\&A, 263, 292
Eislöffel, J., \& Mundt, R. 1997, AJ, 114, 280

Fazio, G., et al. 2004, ApJS, 154, 10

Ferreira, J. 2008, New Astron. Rev., 52, 42

García, P. J. V., \& Ferreira, J. 2010, Jets from Young Stars IV: From Models to Observations and Experiments (Lecture Notes in Physics, Vol. 793; Berlin: Springer)

García López, R., Nisini, B., Giannini, T., Eislöffel, J., Bacciotti, F., \& Podio, L. 2008, A\&A, 487, 1019

Heathcote, S., \& Reipurth, B. 1992, AJ, 104, 2193

Herbig, G. H. 1974, Lick Obs. Bull., 658, 1

Looney, L. W., Tobin, J. J., \& Kwon, W. 2007, ApJ, 670, 131

Menten, K. M., Reid, M. J., Forbrich, J., \& Brunthaler, A. 2007, A\&A, 474, 515

Morse, J. A., Hartigan, P., Cecil, G., Raymond, J. C., \& Heathcote, S. 1992, ApJ, 399, 231

Morse, J. A., Heathcote, S., Hartigan, P., \& Cecil, G. 1993, AJ, 106, 1139

Noriega-Crespo, A., Moro-Martín, A., Carey, S., Morris, P. W., Padgett, D. L., Latter, W. B., \& Muzerolle, J. 2004, ApJS, 154, 352

Reipurth, B., Bally, J., Graham, J. A., Lane, A. P., \& Zealy, W. J. 1986, A\&A, 164,51

Reipurth, B., Heathcote, S., Morse, J., Heathcote, S., \& Bally, J. 2002, AJ, 123, 362

Reipurth, B., Yu, K. C., Heathcote, S., Bally, J., \& Rodríguez, L. F. 2000, AJ, 120,1449

Rieke, G. H., et al. 2004, ApJS, 154, 25

Rodríguez, L. F., Delgado-Arellano, V. G., Gómez, Y., Reipurth, B., Torrelles, J. M., Noriega-Crespo, A., Raga, A. C., \& Cantó, J. 2000, AJ, 119, 882

Stanke, T., McCaughrean, M. J., \& Zinnecker, H. 1998, A\&A, 332, 307

Stapelfeldt, K. R., \& Scoville, N. Z. 1993, ApJ, 408, 239

Stapelfeldt, K. R., Scoville, N. Z., Beichman, C. A., Hester, J., \& Gauthier, T. 1991, ApJ, 371, 226

Tobin, J. J., Looney, L. W., Mundy, L. G., Kwon, W., \& Hamidouche, M. 2007, ApJ, 659, 1404

Werner, M. W., et al. 2004, ApJS, 154, 1

Ybarra, J. E., \& Lada, E. A. 2009, ApJ, 695, 120 